\title{
Boom time for biomaterials
}

\author{
Rapid developments are afoot in the field of biomaterials, and are likely to have major effects on patient \\ care soon. But science isn't the only thing defining the pace of progress.
}

In this issue of Nature Materials we take a look at recent advances in the field of biomaterials - the study of interactions between in vivo or in vitro biological systems and materials - and look at what we can expect in the future. The move of modern biomaterials from lab to hospital may seem to be a rapid one, but humans have been augmenting the body with implants for centuries. As far back as $200 \mathrm{AD}$ it is thought that iron dental implants were used in Europe, and the Mayans fashioned nacre teeth from sea shells around $600 \mathrm{AD}$; both types of material became integrated into the bone after implantation ${ }^{1}$.

Although what constitutes the very first biomaterial may remain somewhat illdefined, it is clear that modern biomaterials are evolving at an intense pace. From simple implants like intraocular lenses, which restore sight to millions of cataract patients every year and were first used more than half a century ago, to more complex materials that not only perform mechanical tasks in the body, but can also interact with and even direct the body's response for maximum restorative power. Indeed, researchers are becoming adept at creating grafts and implants that don't only mimic the body, but actually encourage it to 'colonize' the foreign material itself, growing new skin, cartilage or blood vessels. This boom is in part a result of more specific knowledge about the human body at the cellular level. The improved understanding of body tissue and its interaction with materials, together with the increase in collaboration from scientists from different disciplines to help disseminate this knowledge and enable its use in engineering, has allowed the rapid developments that we are now seeing.

The step changes in the approach to biomaterials are examined in our special issue: the evolution of biomaterials from simple embedded devices to more complex functional materials that control biological interactions is examined in our Commentary from James Burns, who argues that it is likely to be the most prosperous research and development direction in the future for the field ${ }^{2}$. And developments in the field of tissue engineering are reviewed by Molly Stevens and colleagues ${ }^{3}$, who are of the opinion that the field is better served by trying to generate less complex materials that can stimulate the

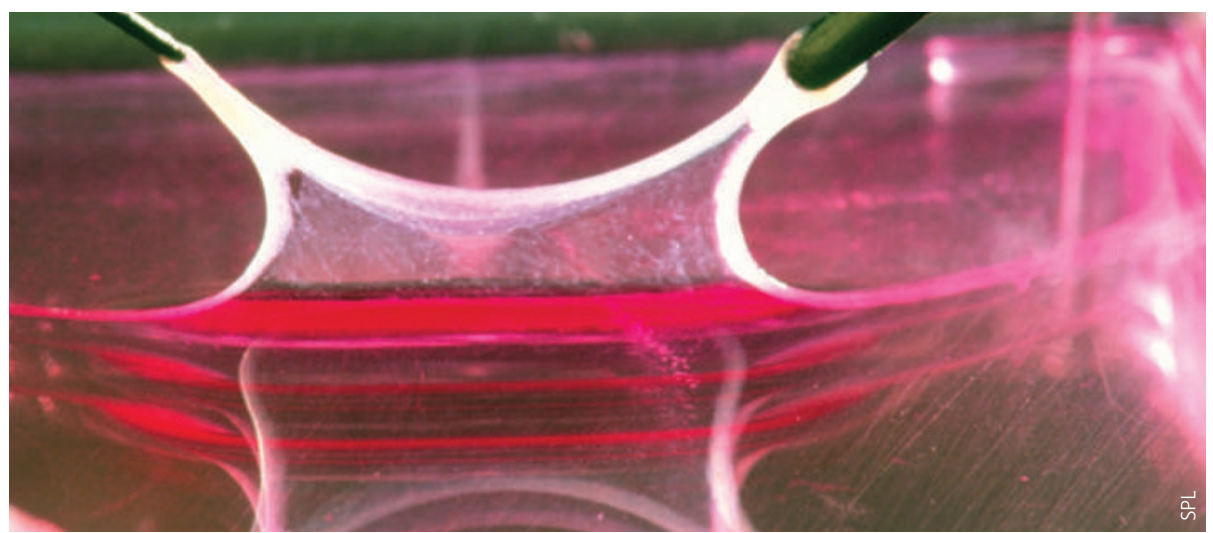

Artificial human epidermis in a liquid-culture flask; new skin grafts grown from patients' own cells have lower risks of rejection.

body to heal and improve itself using biology as a tool, rather than concentrating on more and more complex materials to replace body tissues themselves.

A field such as biomaterials touches on many ethical questions, from sample sources to how advances are deployed into clinical care across the population. The wider public, politicians and policy makers quite rightly have a role in considering these questions, resulting in research in this area regularly being scrutinized and influenced by external factors. For instance, a current research area in biomaterials focuses on how materials properties, such as mechanical elasticity or chemical properties, can influence the differentiation and growth of cells; indeed, this is a common theme for papers within our own pages ${ }^{4,5}$. For this type of study, stem cells are of particular use, as they begin as unspecialized cells with the ability to grow into different types of cell depending on need. The well-publicized ethical debate surrounding the sourcing of stem cells from embryos has been going on for some years, and is not limited to those that are used in biomaterials studies.

On 9 March 2009, President Obama lifted restrictions laid down by the Bush administration on federal funding for research into new stem cell lines. A single embryo can provide a theoretically limitless source of stem cells as the line can be grown indefinitely. However, researchers have been in need of stem cell lines with more diverse genetic make up, including those with genetic problems such as Parkinson's and autoimmune diseases. Obama's move has freed US scientists to add to the currently available 21 stem cell lines, broadening research possibilities into specific genetic problems. Clearly, these political and ethical debates are needed, but some US researchers felt that the Bush administration's stance put them at a disadvantage compared with their colleagues in other countries ${ }^{6}$. Legal factors, such as the fear of litigation mentioned by Robert Langer in our interview ${ }^{7}$, will also have a role in what research scientists and companies are willing and able to undertake and fund.

Back in the biomaterials research lab, the multidisciplinary approach that is helping to fuel growth fits perfectly with the ethos of materials science in general, and reflects the tactics used by a large number of our authors and readers. We look forward to seeing what happens next, and hope that science, not external factors, will be the major determinant of the nature of future research and the speed with which these developments occur.

\footnotetext{
References

1. Ratner, B. D. et al. Biomaterials Science: An Introduction to Materials in Medicine (Academic Press, 2004).

2. Burns, J. W. Nature Mater. 8, 441-443 (2009).

3. Place, E. S., Evans, N. D. \& Stevens, M. M. Nature Mater. 8, 457-470 (2009).

4. Mitragotri, S. \& Lahann, J. Nature Mater. 8, 15-23 (2009).

5. Benoit, D. S. W., Schwartz, M. P., Durney, A. R. \& Anseth, K. S. Nature Mater. 7, 816-823 (2008).

6. Owen-Smith, J. \& McCormick, J. Nature Biotech. 24, 391-392 (2006). 7. Nature Mater. 8, 444-445 (2009).
} 